\title{
COVID-19: General and Oral Manifestations in Children, Impact on Pediatric Dentistry and Future Perspective
}

\author{
Barun Kumar Sah, ${ }^{1}$ Sunanda Sundas, ${ }^{2}$ Neha Dhakal ${ }^{3}$ \\ 1,3 Assistant Professor, ${ }^{2}$ Associate Professor, \\ Department of Pediatric and Preventive Dentistry, People's Dental College and Hospital, Kathmandu, Nepal.
}

\begin{abstract}
Coronavirus disease 2019 (COVID-19) has spread rapidly across the globe, becoming a major public health concern for countries around the world. Despite worldwide efforts to contain viral spread, the outbreak has not shown any promising signs of containment yet. The coronavirus disease 2019 is a respiratory infectious disease caused by severe acute respiratory syndrome coronavirus- 2 (SARS-CoV-2). It is mainly transmitted through respiratory droplets and close contact. As COVID-19 cases have been reported among children as well, there is an increased risk of acquiring the disease through these Silent Carriers who present as asymptomatic with mild or moderate clinical viral infection. The American Academy of Pediatric Dentistry (AAPD) is constantly revising their checklist for re-emergence in pediatric dental practice during the COVID-19 pandemic. Pediatric dentists should keep a high level of awareness to help patients, minimize risk and prevent viral spread. During this period of pandemic, management of child's oral health presents specific difficulties related to the infectious spread of the disease. The aim of this article is to review the Covid-19 infection in children, its impact on pediatric oral health, challenges for pediatric dentistry and future perspective.
\end{abstract}

Keywords: COVID-19, impact, oral manifestations, pandemic, pediatric dentistry.

\section{INTRODUCTION}

The novel coronavirus was first identified in Wuhan, China in December 2019 where the patient presented with pneumonia of unknown origin. The World Health Organization (WHO) on 9th January 2020, declared the discovery of a new coronavirus, first called 2019-nCoV and then officially named SARS-CoV-2, which had never been identified in humans before. The respiratory disease deriving from SARS-CoV-2 infection was named COVID-19 on 11th February 2020. ${ }^{1}$ Coronavirus disease 2019 (COVID-19) has spread rapidly across the globe, becoming a major public health challenge for countries around the world. ${ }^{2}$ COVID-19 is a Severe Acute Respiratory Syndrome (SARS) caused by SARS-CoV-2 virus. $^{3}$ The causative agent is a positive-stranded RNA

\section{Correspondence}

Dr. Barun Kumar Sah.

Assistant Professor, Department of Pediatric and Preventive

Dentistry, People's Dental College and Hospital, Kathmandu, Nepal

Email: drbarunsh@gmail.com

\section{Citation}

Sah BK, Sundas S, Dhakal N. COVID-19: General and Oral Manifestations in Children, Impact on Pediatric Dentistry and Future Perspective. J Nepalese Assoc Pediatr Dent. 2020;1(1):39-43. virus which gains entry into the host cell by attaching itself to the angiotensin-converting enzyme 2 (ACE 2). ${ }^{4}$

The possible routes of transmission of COVID-19 include inhalation of airborne microorganisms that can remain suspended in air for long periods; direct contact with blood; contact of conjunctival, nasal or oral mucosa with droplets and aerosols containing microorganisms generated from an infected individual and propelled by coughing, sneezing and talking; indirect contact with contaminated surfaces. ${ }^{5}$ However, the affected patients also present with abdominal discomfort and the gastrointestinal system has also been identified as a potential route of transmission. ${ }^{4}$

SARS-CoV-2 is a new and an unpredictable virus which has rapid human to human transmission, and unfortunately as of today, there is no effective medication available to halt the rapid progression of the disease and vaccine is on its way and its promising. This virus has gone on to cause one of the most rapidly expanding pandemics we have known since the Spanish Flu pandemic in the early 1900s, with over 9 million people being infected worldwide including the pediatric population. ${ }^{6}$

By imposing a nationwide lockdown, Nepal has curtailed the spread of this virus to a certain extent; however, the total number of reported cases as of 25th November, 2020 
has reached 224077 with approximately 1361 deaths and these numbers are rising every day. The total number of COVID-19 cases in age group from 0-14 years is 10257 with 11 deaths. $^{7}$ The COVID-19 disease has a profound impact on daily lives, with over one-third of the global population in lockdown as the world battles to contain the pandemic.

The guidance on COVID-19 is constantly being updated because of the novel nature of this disease. The impact of COVID-19 on dentistry has been profound due to the way in which the virus spreads through aerosol. As a result of which, those in the dental profession especially the pediatric dentist are at high risk of contacting and spreading the virus to the general population through aerosol generating procedures (AGPs). ${ }^{8}$ The pediatric dentists are in very close vicinity of the child during behavior management and also due to the very presence of caregivers in the dental operatory, further increases the risk of infection. ${ }^{9}$

In this current scenario, the aerosol generating procedures have to be replaced with a more minimally invasive or nonaerosol generating ones which include use of SDF, ART, chemomechanical caries removal and Hall technique along with the use of preventive measures like sealants, remineralizing agents and resin infiltration technique. ${ }^{10}$ The home care measures taken up by parents to maintain a proper oral hygiene at home helps to better enhance the overall oral health. The literature concerning the impact of COVID-19 on pediatric dentistry is quite limited. Hence, this review article focuses on COVID-19 pandemic, its impact on pediatric oral health, challenges for pediatric dentistry and future perspective from pediatric dentistry point of view.

\section{COVID-19 AND ITS GENERAL MANIFESTATION IN CHILDREN}

The estimated incubation period of the disease COVID-19 ranges from 1-14 days, which is also the duration of medical observation and quarantine in exposed patients. ${ }^{1}$ Pediatric COVID-19 infection is reported to be relatively mild in symptoms when compared to adults and adolescents. A large proportion of infected children appears to be asymptomatic and may contribute significantly to transmission. A specific concern for children is the uncertainty of their infection status; a clinical history may not be as suggestive of the infection as it is in adults. ${ }^{3}$
The symptoms include fever, dry cough, and fatigue, with a few upper respiratory symptoms like nasal congestion and runny nose. It rarely progresses downward to involve lower respiratory tract. Some of the obscure symptoms like nausea, diarrhea, hyposmia, dysguesia, abdominal discomfort, vomiting have also been reported. The recovery period is within 1 to 2 weeks after the onset in children. The positive aspect of COVID-19 infection in children is the good prognosis with a fatality rate of $0.01 \%$ despite having wide range of clinical manifestations. ${ }^{10,11}$

The first pediatric case in the United States was reported on 2nd March, 2020. The children below the age of 18 who have tested positive, represents around $1.7 \%$ of the total infected population with no significant gender predilection. ${ }^{12}$ It has been reported that human coronavirus infections may also involve central nervous system. ${ }^{13}$

The Chinese Centers for Disease Control and Prevention reports that of the 72,314 cases reported as of 11th February, 2020, only 2\% were in individuals of less than 19 years of age. The data suggests that children are less commonly affected by COVID-19. From a small case series of 9 mothers who were infected with SARS-CoV-2, there is, to date, no evidence that SARS-CoV-2 can be vertically transmitted to the infant. ${ }^{14}$ According to Cruz and Zeichner (2020), only 4\% of virologically confirmed cases had asymptomatic infection, and this rate almost certainly understates the true rate of asymptomatic infection because many children who are asymptomatic are unlikely to be tested. The preschool-aged children and infants have severe clinical manifestations than older children. ${ }^{15}$

The reason is still confusing regarding less severity of COVID-19 cases in children than in adults. However, more active innate immune response, healthier respiratory, and less underlying illnesses may be the possible explanations. The weaker ability to trigger an acute inflammatory response to SARS-CoV-2 might also contribute to the children's better outcome. ${ }^{2}$

\section{ORAL MANIFESTATIONS OF COVID-19 IN CHILDREN}

COVID19 also has oral manifestations as do many other viral infections. ${ }^{16}$ The oral manifestations include salivary gland infection, taste abnormalities, oral ulceration, blistering and dry mouth. ${ }^{17}$ The oral cavity may be a perfect 
habitat for SARS-CoV-2 invasion due to its special affinity for cells with the receptors for the converted angiotensin enzyme (ACE2) that is present in the respiratory tract, oral mucosal, tongue and salivary glands. As a result of which, it is able to affect the functioning of the salivary glands, sensations of taste, smell and integrity of the oral mucosa. It could have the ability to alter the balance of the oral microbiota, which added to a depressed immune system would allow opportunistic infections colonization. ${ }^{18}$

Saliva can have a pivotal role in the human-to-human transmission. Sabino-Silva et al. suggested that there is a minimum of three different pathways for COVID-19 to be present in saliva: from COVID-19 in the lower and upper respiratory tract, that enters the oral cavity together with the liquid droplets frequently exchanged by these organs; COVID-19 present in the blood can access the mouth via crevicular fluid; by major- and minor-salivary gland infection, with subsequent release of particles in saliva via salivary ducts. ${ }^{19}$

The milder form of COVID-19 infection in children have usually non- specific intraoral findings. Martin et al in 2020 presented a case series where ulcers and blisters were seen as oral manifestations in affected or suspected cases. ${ }^{20}$ There is no literature yet available that states the intraoral findings in a COVID positive child so the pediatric dentists must be vigilant while examining COVID positive children due to the lack of adequate information. ${ }^{10}$ However, one of the study supports the evidence that oral ulcerations and vesiculobullous lesions are not manifestations and dysgeusia is the only oral symptom of COVID-19 as reported in the current literature. So the dentists must be aware of this information whose treatment decisions may be impacted under the erroneous assumption that oral ulcerations or blisters are manifestations of COVID-19. ${ }^{21}$

\section{IMPACT ON PEDIATRIC DENTISTRY}

The whole of the dental fraternity have been hit hard by the coronavirus pandemic. Pediatric dentistry is in a unique position in this period. Most of the dental organizations and governing body suggests to perform only emergency dental procedures and pediatric dentistry is no different. The American Academy of Pediatric Dentistry (AAPD) has been regularly updating about treatment guidelines that advises pediatric dentists to postpone all elective procedures for at least three weeks but to continue emergency or urgent care. They also suggest that elective general anesthesia cases be postponed so that operating room resources are not stressed. ${ }^{4}$

In pediatric dental practice, effective infection control measures for the prevention or minimization of viral infection transmission can be implemented by: a) controlling the gag or cough reflex b) reducing aerosol/ splatter generation c) managing contaminated air and d) improving personal protection. Since the children and adolescents passes through the different stages of physical, intellectual, emotional and social development, they present major challenges for pediatric dentists during dental procedure especially attending to their psychological needs within adequate infection control regimen. ${ }^{13}$

Due to COVID-19 pandemic, there is massive change in the working atmosphere which include use of personal protective equipment (PPE), face shield, sanitizer, disinfectant and other additional safety measures that have further increased the financial burden. On top of that, managing the pediatric patients with PPE further makes the working environment more challenging and limits the number of cases to be treated as well. It has greater negative impact on the newcomer pediatric dentists who have just started their private practice or are on the verge of initiating their career. Also the government has issued the guidelines that further restricts the movement of the children and at the same time parents are reluctant to bring them for the dental treatment. It cannot be denied that the parents, the children and the pediatric dentists all are in the constant fear of contacting the disease.

COVID-19 has disrupted the conventional way of practicing and teaching pediatric dentistry in the United States. In the United States, all children are currently treated as suspected to have COVID-19 until there is valid and rapid testing of children and parents. ${ }^{12}$ The unpredictability of COVID-19 infection in children and the challenges in the diagnosis of symptoms, in addition to the reports of carrier status of the infection will present a challenge for all pediatric dentists once normal function in the dental clinics is restored worldwide which may result in increased anxiety for the dental healthcare providers, the parents and the patients. The skillful behavior management technique is one of the way to minimize the possibility of cross-infection. ${ }^{3}$

It is futile to imagine that COVID-19 will just suddenly disappear; rather, it is likely to remain an issue in the long 
term with a second wave of COVID-19 cases already being reported in some countries. So the alternative to conventional dental treatment of the carious primary teeth should be considered such as Minimal Intervention Dentistry (MID), sealants, resin infiltration, silver diammine fluoride (SDF) application, the Hall Technique, Atraumatic Restorative Technique (ART), and selective removal of carious tissue to soft and firm dentine. ${ }^{6}$

The role of dental professionals in preventing the transmission of COVID-19 is critically important since it has the most risk of spreading the virus than any profession in relation to COVID-19.22 The mild symptoms of the infection among the children, the possible aerosol transmission, and the incubation period without symptoms prompt the dentists to take certain precautions to minimize the risk of the transmission in the dental office. ${ }^{23}$ The use of handpiece should be limited and if possible, dental procedures should be carried out with manual instruments under rubber dam isolation and surgical aspiration to limit aerosol diffusion. The time duration of health care contact with patients should be limited to 15 minutes as per suggestion of the Italian National Institute of Health to reduce the risk of contact $^{1}$ which seems to be practically challenging job in exclusive pediatric dentistry practice.

\section{FUTURE PERSPECTIVE}

The countries all-round the globe are responding in their own way to combat this COVID-19 pandemic. Most of the country have imposed nationwide lockdown and issued various guidelines in response to the pandemic. The elderly people, the children below the age of 14 years and those with underlying co-morbidities are considered to be at high risk.

The pandemic has overburdened the health care system of the country further deteriorating the economic crisis. The health care system and the responsible bodies should take this challenges as a golden opportunity to learn from the current experience of this pandemic to make plan for the future design of the optimal health care system that enhance quality care, accessibility and minimum burden of cost on the patient. ${ }^{17}$ Social distancing, improving personal hygienic practices and use of mask in the public places are considered as new normal. These are the major public health interventions effective in delaying the widespread community transmission, reducing peak incidence and its impact on public services and decreasing the overall attack rate. ${ }^{24}$
The current pandemic has the potential to change the dental landscape for the foreseeable future. Consideration needs to be given to potential vaccine development, the likelihood of a 'second peak' and the ever-changing evidence-based guidelines which highlight the impact COVID-19 is having upon both dental practitioners and patients. ${ }^{8}$ The Italian Society of Pediatric Dentistry (SIOI) has advised, to take minimally invasive dentistry (MID), atraumatic restorative treatment, use of fissure sealants, silver diamine fluoride (SDF), selective caries removal and the Hall Technique, into consideration until COVID-19 will be completely eliminated. ${ }^{2}$

With a paradigm shift in dental care practice in progress during the current pandemic situation, teledentistry holds the prospects to attend the treatment needs of the patients without confrontation. ${ }^{25}$ The teledentistry satisfies the need for social distancing as has been advocated by the health authorities all across the globeto contain the spread disease.It can be incorporated into routine dental practice as it offers a wide range of applications such as remote triaging of the suspected COVID-19 patients for dental treatment and decreasing the unnecessary exposure of healthy or uninfected patients by decreasing their visits to already burdened dental offices and hospitals. The teledentistry can complement the existing compromised dental system during the current pandemic. ${ }^{26}$

Once practice restrictions begin to be eased, continued management of dental disease with contemporary dentistry through minimally interventive concepts and other non-AGPs, whilst viral transmission risk remains high, will be pertinent. The importance of tooth-brushing with fluoridated dentrifice to prevent dental caries should continue to be emphasized during contact with patients, and there are opportunities being taken for dentists to carry out telephone and video consultations with parents to promote positive oral health behaviors. ${ }^{27}$

Unfortunately, the pandemic COVID-19 will not stop immediately and everyone will have to face each other very long working and social recovery times of the population. In this time, a large part of the population will avoid dental treatment other than those imposed by pain or urgency, both due to money issues and, principally, for a psychological reason: it will not be easy to overcome the fear of infection. ${ }^{28}$ 


\section{CONCLUSION}

COVID-19 has and will continue to have major impacts on the practice of pediatric dentistry across the globe. COVID-19 seems to affect children less severely as compared to adults, they still remain vulnerable to infection and pose a substantial transmission risk. Pediatric dentists during this pandemic should enact universal infection control protocols to the highest standards to limit the spread. Once the current worldwide restrictions are eased, we might need to modify some approaches in our regular practice of pediatric dentistry in order to minimize the risk of spreading the infection to patients, dental staff, and public posed by the dental procedures.

\section{Conflict of Interest: None}

\section{REFERENCES}

1. Izzetti R, Nisi M, Gabriele M, Graziani F. COVID-19 Transmission in Dental Practice: Brief Review of Preventive Measures in Italy.J Dent Res. 2020 Aug;99(9):10301038. [PubMed I DOI]

2. Ferrazzano GF, Ingenito A, Cantile T. COVID-19 Disease in Children: What Dentists Should Know and Do to Prevent Viral Spread. The Italian Point of View. In J Environ Res Public Health. 2020 May 22;17(10):3642. [PubMed I DOI]

3. Al-Halabi M, Salami A, Alnuaimi E, Kowash M, Hussein I. Assessment of paediatric dental guidelines and caries management alternatives in the post COVID-19 period. A critical review and clinical recommendations. Eur Arch Paediatr Dent. 2020 Oct;21(5):543-556. [ubMed I DOI]

4. $\quad$ Singh V, Lehl GK, Talwar M, Luthra A. The novel coronavirus and challenges for general and paediatric dentists. Occup Med (Lond). 2020 Jul 17;70(5):320-322. [PubMed | DOI]

5. Peng X, Xu X, Li Y, Cheng L, Zhou X, Ren B. Transmission routes of 2019-nCoV and controls in dental practice. Int J Oral Sci. 2020 Mar 3;12(1):9. [PubMed I DOI

6. BaniHani A, Gardener C, Raggio DP, Santamaría RM, Albadri S. Could COVID-19 change the way we manage caries in primary teeth? Current implications on Paediatric Dentistry. Int J Paediatr Dent. 2020 Sep;30(5):523-525. [ubMed I DOI]

7. Situation Update \#32 - Coronavirus Disease 2019 (COVID-19) WHO Country Office for Nepal Wednesday 25 November 2020. Retrieved 11 December 2020. Available from: https://www.who.int/docs/default-source/nepal-documents/novel-coronavirus/who-nepal-sitrep/-32_who_nepal_sitrep_covid-19.pdf?sfvrsn=4774d84_5. [Full Text]

8. Ilyas N, Agel M, Mitchell J, Sood S. COVID-19 pandemic: the first wave - an audit and guidance for paediatric dentistry. Br Dent J. 2020 Jun;228(12):927-931. PubMed I DOI]

9. Luzzi V, Ierardo G, Bossù M, Polimeni A. Paediatric Oral Health during and after the COVID-19 Pandemic. Int J Paediatr Dent. 2021 Jan;31(1):20-26. [PubMed I DOI]

10. Koticha PB, Pradhan D, Katge F, Krishna V, Bhanushali P, Patil D. COVID-19 in children: its impact on oral health and paediatric dentistry. Int J Sci Healthc Res. 2020;5(3):377-89. [Full Text]

11. Hong H, Wang Y, Chung HT, Chen CJ. Clinical characteristics of novel coronavirus disease 2019 (COVID-19) in newborns, infants and children. Pediatr Neonatol. 2020 Apr;61(2):131-132. [PubMed I DOI]

12. Jayaraman J, Dhar V, Moorani Z, Donly K, Tinanoff N, Mitchell S, Wright T. Impact of COVID-19 on Pediatric Dental Practice in the United States. Pediatr Dent. 2020 May 15;42(3):180-183. [PubMed]

13. Al-Nerabiah Z, Alkhouli M, Laflouf M, Abdul-Hak M. Pediatric dentists consideration for Covid-19 in children: Review article. International Journal of Applied Dental Sciences. 2020; 6(2): 628-630. [Full Text]

14. Zimmermann P, Curtis N. Coronavirus Infections in Children Including COVID-19: An Overview of the Epidemiology, Clinical Features, Diagnosis, Treatment and Prevention Options in Children. Pediatr Infect Dis J. 2020 May;39(5):355-368. [PubMed I DOI]

15. Cruz AT, Zeichner SL. COVID-19 in Children: Initial Characterization of the Pediatric Disease. Pediatrics. 2020 Jun;145(6):e20200834. [PubMed I DOI]

16. Vieira AR. Oral manifestations in coronavirus disease 2019 (COVID-19). Oral Dis. 2020 Jun 7:10.1111/odi.13463. [PubMed | DOI]

17. Pant, B. (2020). Covid-19: Oral manifestations, impact and lessons for the future. Orthodontic Journal of Nepal, 10(2), 52-54. [Full Text I DOD]

18. NEMETH, K. M. E.; MATUS, A. C. P. \& CARRASCO, S. R. R. Oral manifestations of COVID-19. Int. J. Odontostomat., 14(4):555-560, 2020. Retrieved 15 September 2020. Available from: http:/www.ijodontostomatology.com/en/articulo/oral-manifestations-of-covid-19/ [Full Text I Link]

19. Sabino-Silva R, Jardim ACG, Siqueira WL. Coronavirus COVID-19 impacts to dentistry and potential salivary diagnosis. Clin Oral Investig. 2020 Apr;24(4):1619 1621. [PubMed I DOI]

20. Martín Carreras-Presas C, Amaro Sánchez J, López-Sánchez AF, Jané-Salas E, Somacarrera Pérez ML. Oral vesiculobullous lesions associated with SARS-CoV-2 infection. Oral Dis. 2020 May 5:10.1111/odi.13382. [uMed I DOI]

21. Al-Khatib A. Oral manifestations in COVID-19 patients. Oral Dis. 2020 Jun 10:10.1111/odi.13477. [PubMed I DOI]

22. Shah S. COVID-19 and paediatric dentistry- traversing the challenges. A narrative review. Ann Med Surg (Lond). 2020 Oct;58:22-33. [ubMed I DOI]

23. Bahramian H, Gharib B, Baghalian A. COVID-19 Considerations in Pediatric Dentistry. JDR Clin Trans Res. 2020 Oct;5(4):307-311. [PubMed I DOI]

24. Wilder-Smith A, Chiew CJ, Lee VJ. Can we contain the COVID-19 outbreak with the same measures as for SARS? Lancet Infect Dis. 2020 May;20(5):e102-e107. [PubMed I DOI]

25. Bhanushali P, Katge F, Deshpande S, Chimata VK, Shetty S, Pradhan D. COVID-19: Changing Trends and Its Impact on Future of Dentistry. Int J Dent. 2020 May 29;2020:8817424. [PubMed I DOI]

26. Ghai S. Teledentistry during COVID-19 pandemic. Diabetes Metab Syndr. 2020 Sep-Oct;14(5):933-935. [PubMed I DOI]

27. Mallineni SK, Innes NP, Raggio DP, Araujo MP, Robertson MD, Jayaraman J. Coronavirus disease (COVID-19): Characteristics in children and considerations for dentists providing their care. Int J Paediatr Dent. 2020 May;30(3):245-250. [PubMed I DOI]

28. Bizzoca ME, Campisi G, Muzio LL. Covid-19 Pandemic: What Changes for Dentists and Oral Medicine Experts? A Narrative Review and Novel Approaches to Infection Containment. Int J Environ Res Public Health. 2020 May 27;17(11):3793. [PubMed | DOI] 